\title{
AN ASYMPTOTIC FORMULA FOR BOUNDARY POTENTIAL PERTURBATIONS IN A SEMILINEAR ELLIPTIC EQUATION RELATED TO CARDIAC ELECTROPHYSIOLOGY
}

\author{
ELENA BERETTA* \\ Dipartimento di Matematica, Politecnico di Milano, P.za Leonardo da Vinci 32, \\ Milano, I-20133, Italy \\ elena.beretta@polimi.it \\ M. CRISTINA CERUTTI \\ Dipartimento di Matematica, Politecnico di Milano, P.za Leonardo da Vinci 32, \\ Milano, I-20133, Italy \\ cristina.cerutti@polimi.it \\ ANDREA MANZONI \\ MATHICSE-CMCS-SB, Ecole Polytechnique Fédérale de Lausanne, Station 8, \\ Lausanne, CH-1015, Switzerland \\ andrea.manzoni@epfl.ch \\ DARIO PIEROTTI \\ Dipartimento di Matematica, Politecnico di Milano, P.za Leonardo da Vinci 32, \\ Milano, I-20133, Italy \\ dario.pierotti@polimi.it
}

\begin{abstract}
In this paper we provide a representation formula for boundary voltage perturbations caused by internal conductivity inhomogeneities of low volume fraction in a simplified monodomain model describing the electrical activity of the heart. We derive such a result in the case of a nonlinear problem. Our long-term goal is the solution of the inverse problem related to the detection of regions affected by heart ischemic disease, whose position and size are unknown. We model the presence of ischemic regions in the form of small inhomogeneities. This leads to the study of a boundary value problem for a semilinear elliptic equation. We first analyze the well-posedness of the problem establishing some key energy estimates. These allow us to derive rigorously an asymptotic formula of the boundary potential perturbation due to the presence of the inhomogeneities, following an approach similar to the one introduced by Capdeboscq and Vogelius in ${ }^{9}$ in the case of the linear conductivity equation. Finally, we propose some ideas of the reconstruction procedure that might be used to detect the inhomogeneities.
\end{abstract}

Keywords: elliptic semilinear equation; asymptotic expansion; inverse problem

AMS Subject Classification: 35J65, 35R30, 35R05, 35C20

${ }^{*}$ Corresponding Author 


\section{Introduction}

Ischemic heart disease results from a restriction in blood supply to the heart and represents the most widespread heart disease. As a consequence, myocardial infarction (or heart attack) caused by the lack of oxygen might lead to even more severe heart muscle damages, ventricular arrhythmia and fibrillation, ultimately causing death. Detecting ischemic heart diseases - that is, recovering the unknown shape (and/or position) of ischemic areas - at early stages of their development from noninvasive (or minimally invasive) measurements is thus of primary importance.

This is usually performed by recording the electrical activity of the heart, by means of either body surface or intracardiac measurements. In the former case, the electrocardiogram (ECG) records electrical impulses across the thorax, by means of a set of electrodes attached to the surface of the skin. In the latter case, intracardiac electrograms, that is, measurements of intracavitary potentials, are obtained by means of non-contact electrodes carried by a catheter inside a heart cavity. Although much more invasive than ECG, this latter technique has become a standard of care in patients with symptoms of heart failure, and allows to get a map of the endocardial potential.

In this context, mathematical models could be used to shed light on the potentialities of electrical measurements in detecting ischemias. More specifically, the goal would be to combine measurements of (body-surface or intracavitary) potentials and a mathematical description of the electrical activity of the heart in order to identify the position, the shape and the size of heart ischemias and/or infarctions. It is well known (see e.g. ${ }^{20,11}$ ), that a mathematical description of the electrical activity of the heart is provided by the so-called bidomain model, yielding to an initial boundary value problem for a coupled nonlinear evolution system. A simplified, one-field version of this problem is provided by the monodomain model, resulting in a nonlinear diffusion-reaction equation. Moreover, the myocardium is surrounded by a volume conductor, the torso, which is commonly modeled as a passive conductor through a linear elliptic equation; heart and torso are coupled by imposing the continuity of the electrical potential and the currents across the interface.

The challenge of how to combine ECG recordings or intracavitary potential measurements with numerical simulations to identify ischemic heart disease has by far not been investigated enough. Although some analysis of the direct problem has been carried out, - see, e.g. ${ }^{6}$ - and such a model (coupled with the torso) has been exploited for the ultimate generation of synthetic ECG data ${ }^{5}$, to our knowledge there is no theoretical investigation of inverse problems related with ischemia detection involving the monodomain and/or the bidomain model, not even 
in the case of an isolated heart. In the past decade some numerical investigations dealing with ischemia identification from measurements of surface potentials have been performed by casting the problem in an optimization framework. A stationary model taking into account the heart-torso coupling has been employed in ${ }^{17}$, whereas a nonstationary monodomain model for an isolated heart has been considered in ${ }^{16}$. More recently, the case of ischemias identification from intracardiac electrograms has been treated in ${ }^{2}$.

The question of finding the ischemic region can be formulated as the inverse problem of detecting inhomogeneities, whose position and size are unknown, in a nonlinear parabolic diffusion-reaction PDE modeling (for the time being, a much simplified version of) the cardiac electrical activity, from boundary measurements ${ }^{\mathrm{a}}$. In the present paper we assume to be able to perform measurements on the heart (by one of the devices described above) and use an insulated monodomain model in the steady state. This leads to the study of a Neumann boundary value problem for a semilinear elliptic equation.

We assume that the ischemic region is a small inclusion $\omega_{\epsilon}$ with a significantly different conductivity from the healthy tissue. Taking advantage of the smallness of the inclusion, we establish a rigorous asymptotic expansion of the boundary potential perturbation due to the presence of the inclusion following the approach introduced by Capdeboscq and Vogelius in ${ }^{9}$ for the case of the linear conductivity equation. It turns out that this approach has been successfully used for the reconstruction of location and geometrical features of the unknown inclusions from boundary measurements ${ }^{3,4}$ in the framework of Electrical Impedance Tomography (EIT) imaging techniques. Despite of the fact that we have to deal with a nonlinear equation, we derive a rigorous expansion for the perturbed electrical potential and give also some idea of the reconstruction procedure that might be used to detect the inclusion.

The paper is organized as follows: in Section 2 we illustrate the monodomain model for the cardiac electrical activity and motivate the assumptions at the basis of our simplified model. In Section 3 we state our main result. In Section 4 we analyze the wellposedness of the direct problem establishing some key energy estimates. In Section 5 we derive the asymptotic formula for the electrical boundary potential. In Section 6, taking advantage of the asymptotic formula, we highlight some ideas for a possible reconstruction algorithm in a simplified two-dimensional geometry. Numerical simulations relying on the results contained in this paper will be the object of a forthcoming publication.

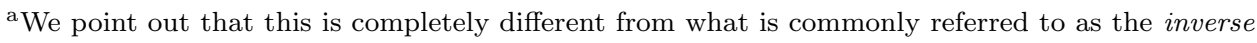
problem of electrocardiography which deals with recovering the electrical potential at the epicardial surface by using recordings of the electrical potential along the body surface, ${ }^{19},{ }^{10},{ }^{21}$ and which involves the pure (linear) diffusion model for the torso as direct problem. 


\section{The direct problem: a nonlinear diffusion-reaction equation}

The bidomain equations are nowadays the most widely accepted mathematical model of the macroscopic electrical activity of the heart ${ }^{20,11}$. This model describes the evolution of the transmembrane (or action) potential, that is, the potential jump $u$ across the cellular membrane surface. Such a model is based on the assumption that at cellular scale cardiac tissue is considered as partitioned in two conducting media, the intracellular (made of cardiac cells) and the extracellular (representing the space between cells) medium, separated by the cell membrane. After a homogenization process, the two media are supposed to occupy the whole heart volume.

The bidomain model consists of a coupled system of time-dependent, nonlinear reaction-diffusion PDEs, whose efficient numerical solution is a very difficult task. Since our main goal is to provide a theoretical analysis of the inverse problem related with the detection of small conductivity inhomogeneities for a nonlinear diffusion-reaction equation, we rather start from the simpler monodomain model. The monodomain model is derived from the bidomain equations by assuming that the extracellular and the intracellular conductivities are proportional quantities. The monodomain model for the electrical activity in the heart in the presence of ischemic regions reads as follows:

$$
\begin{aligned}
\frac{\partial u}{\partial t}-\operatorname{div}\left(k_{\epsilon} \nabla u\right)+\chi_{\Omega \backslash \omega_{\epsilon}} I_{i o n}(u) & =f & & \text { in } Q_{T}=\Omega \times(0, T] \\
\frac{\partial u}{\partial \mathbf{n}} & =0 & & \text { on } \partial \Omega \times(0, T] \\
u(\mathbf{x}, 0) & =u_{0}(\mathbf{x}) & & \text { in } \Omega .
\end{aligned}
$$

where $\Omega$ is the domain occupied by the heart, $u$ is the (transmembrane) electrical potential, $I_{i o n}$ is the ionic membrane current of the heart tissue (up to a capacity constant), $k_{\epsilon}$ is its conductivity, $f$ is an applied current (up to the same capacity constant as $I_{i o n}$ ) and $\chi_{\Omega \backslash \omega_{\epsilon}}$ is the characteristic function of the healthy area. Here $\omega_{\epsilon} \subset \Omega$ is the infarcted area. According to experimental observations, in an ischemic or infarcted region cells are not excitable, so that the conductivity $k_{\epsilon}=k_{\epsilon}(\mathbf{x})$ is substantially different with respect to healthy tissues. For this reason, we define

$$
k_{\epsilon}=\left\{\begin{aligned}
k_{\text {healthy }} \text { in } \Omega \backslash \omega_{\epsilon} \\
k_{\text {infarcted }} \text { in } \omega_{\epsilon}
\end{aligned}\right.
$$

being $k_{\text {infarcted }}=\delta k_{\text {healthy }}$, and $\delta \in(0,1)$. Moreover the ion transport circumvent ischemic areas, so that also the ionic membrane current $I_{i o n}$ is multiplied by $\chi_{\Omega \backslash \omega_{\epsilon}}$ in order to describe a blocking ion transport. In the simplest case, the ionic current $I_{i o n}$ across the cell membrane is assumed to be a nonlinear function of the potential and a significant choice is to assume $I_{i o n}$ to be a cubic polynomial in $u$ such as

$$
I_{\text {ion }}(u)=A^{2}\left(u-u_{\text {rest }}\right)\left(u-u_{\text {th }}\right)\left(u-u_{\text {peak }}\right) .
$$

Here $A>0$ is a parameter determining the rate of change of $u$ in the depolarization phase, and $u_{\text {peak }}>u_{t h}>u_{\text {rest }}$ are given constant values representing the resting, threshold and maximum potentials, which affect the action potential dynamics. 
Equation (2.3) represents the simplest model for the ionic current across the membrane and describes the macroscopic behavior of excitable cells; however, to reproduce the depolarization phase and restitution properties, (2.3) must be extended by introducing a second recovery variable, whose evolution is described by a dynamical system coupled to (2.1); this yields to the so-called Aliev-Panfilov model 1 . More detailed ionic models describe currents across the cell membrane in terms of intra- and extracellular ionic concentration, see e.g. ${ }^{20,11,16}$ and references therein.

Finally, $f$ represents a given current stimulus applied to the tissue - usually in a confined region and for a short time interval - expressing the initial electrical stimulus, related to the so-called pacemaker potential. By solving problem (2.1) we can describe the propagation of the stimulus $f$ in an insulated heart muscle, affected by ischemia in the region $\omega_{\epsilon}$. Changing the size and the location of $\omega_{\epsilon}$ thus results in a different propagation of the applied current.

Starting from model (2.1)-(2.3), we consider some simplifications in the direct problem and hence also in the corresponding inverse problem. In fact, we believe that the key aspect to be tackled is related with the presence of a nonlinear term in the equation. In this paper we consider the steady version of the problem. In this case we replace the term $I_{i o n}=I_{i o n}(u)$ by the cubic nonlinearity $\tilde{I}_{i o n}(u)=u^{3}$ (more generally we could consider a monotone nonlinearity) in order to have uniqueness of the solution to the direct problem. In a forthcoming paper we will analyze the evolutionary problem by considering $I_{\text {ion }}(u)=A^{2}\left(u-u_{\text {rest }}\right)\left(u-u_{t h}\right)\left(u-u_{\text {peak }}\right)$ that results in the bistable behavior of the dynamical system which better describes the electrical potential in the heart. Nonetheless, also in the case where either a recovery variable or more complex ionic models are considered to describe ionic currents, tackling nonlinearities is an essential task which has to be accomplished. Two simplified cases - for which similar results can be shown - are those where only the nonlinear term or the conductivity in the infarcted area are modified; for instance, the infarction is represented through a variation of the coefficient in the ionic model in 17,7 .

\section{Statement of the problem and main result}

As discussed in the previous section, the problem of determining a small 'inhomogeneity' $\omega_{\epsilon}$ inside a smooth domain $\Omega$, meaning a subset in which the conductivity is smaller than in the surrounding tissue leads to solving the following problem for the potential that here will be called $u_{\epsilon}$

$$
\begin{cases}-\operatorname{div}\left(k_{\epsilon}(x) \nabla u_{\epsilon}\right)+\chi_{\Omega \backslash \omega_{\epsilon}} u_{\epsilon}^{3}=f, & \text { in } \Omega \\ \frac{\partial u_{\epsilon}}{\partial \mathbf{n}}=0, & \text { on } \partial \Omega,\end{cases}
$$

where $\Omega \subset \mathbb{R}^{N}, N=2,3$ and $\omega_{\epsilon} \subset \Omega$ is the set of inhomogeneity that we assume to be measurable and separated from the boundary of $\Omega$, meaning that there exist a compact set $K_{0}$ with $\omega_{\epsilon} \subset K_{0} \subset \Omega$ and $d_{0}>0$ such that

$$
\operatorname{dist}\left(\omega_{\epsilon}, \Omega \backslash K_{0}\right) \geq d_{0}>0 \text {. }
$$


Moreover $\left|\omega_{\epsilon}\right|>0 \forall \epsilon$ and $\left|\omega_{\epsilon}\right| \rightarrow 0$ as $\epsilon \rightarrow 0$. By denoting with $\chi_{\omega_{\epsilon}}$ the indicator function of the set $\omega_{\epsilon}$, it is known that there exist a regular Borel measure $\mu$ and a sequence $\omega_{\epsilon_{n}}$, with $\left|\omega_{\epsilon_{n}}\right| \rightarrow 0$, such that

$$
\left|\omega_{\epsilon_{n}}\right|^{-1} \chi_{\omega_{\epsilon_{n}}} d x \rightarrow d \mu
$$

in the weak ${ }^{*}$ topology of the dual of $\mathcal{C}^{0}(\bar{\Omega})$ (see, e.g. ${ }^{8}$ ). Moreover, $\mu$ is a probability measure and by (3.2) its support lies inside the compact set $K_{0}$.

The function $k_{\epsilon}(x)$ represents the conductivity in the two portions of $\Omega$ and is defined as

$$
k_{\epsilon}=\left\{\begin{array}{l}
1, \text { in } \Omega \backslash \omega_{\epsilon} \\
k, \text { in } \omega_{\epsilon},
\end{array}\right.
$$

where we assume $0<k<1$. The potential $U$ for the unperturbed problem satisfies

$$
\left\{\begin{array}{l}
-\Delta U+U^{3}=f, \text { in } \Omega \\
\frac{\partial U}{\partial \mathbf{n}}=0, \quad \text { on } \partial \Omega .
\end{array}\right.
$$

For any given $U \in \mathcal{C}^{1}(\bar{\Omega})$ we introduce the Green function $N_{U}(x, y)$ for the operator $-\Delta+3 U^{2}$ with homogeneous Neumann condition:

$$
-\Delta_{x} N_{U}(x, y)+3 U^{2}(x) N_{U}(x, y)=\delta(x-y) \quad \text { for } x \in \Omega,\left.\quad \frac{\partial N_{U}}{\partial n_{x}}\right|_{\partial \Omega}=0
$$

We are now ready to state our main result.

Theorem 3.1. Let $f \in L^{p}(\Omega)$ for some $p>N$ and assume that $f(x) \geq m>0$ a.e.in $\Omega$. Let $u_{\epsilon}, U$ denote the solutions to (3.1) and (3.5). Then, there exist a sequence $\omega_{\epsilon_{n}}$ with $\left|\omega_{\epsilon_{n}}\right| \rightarrow 0$ satisfying (3.2), (3.3) and a symmetric matrix $\mathcal{M}(x) \in$ $L^{2}(\Omega, d \mu)$ such that, if $w_{\epsilon_{n}}=u_{\epsilon_{n}}-U$,

$$
w_{\epsilon_{n}}(y)=\left|\omega_{\epsilon_{n}}\right| \int_{\Omega}\left((1-k)(\mathcal{M} \nabla U) \nabla_{x} N_{U}+U^{3} N_{U}\right) d \mu(x)+o\left(\left|\omega_{\epsilon_{n}}\right|\right) \quad y \in \partial \Omega,
$$

where $N_{U}(x, y)$ is the solution of (3.6). Moreover $\mathcal{M}(x)$ satisfies

$$
|\xi|^{2} \leq \xi^{T} \mathcal{M}(x) \xi \leq \frac{1}{k}|\xi|^{2} \quad \forall \xi \in \mathbb{R}^{N}
$$

$\mu$ almost everywhere in $\Omega$.

\section{The direct problem}

\subsection{Existence and uniqueness}

In this section we show a well-posedness result for the direct problem (3.1) and the unperturbed problem (3.5).

Theorem 4.1. Assume that $f \in H^{-1}(\Omega)$, the dual space of $H^{1}(\Omega)$. Then problems (3.1) and (3.5) have a unique solution $u_{\epsilon} \in H^{1}(\Omega), U \in H^{1}(\Omega)$ respectively. 
Proof. By multiplying the equation in (3.1) by a test function $\phi$, integrating by parts and using the boundary Neumann condition, we obtain the weak formulation

$$
\int_{\Omega} k_{\epsilon} \nabla u \nabla \phi+\int_{\Omega \backslash \omega_{\epsilon}} u^{3} \phi=\int_{\Omega} f \phi, \quad \forall \phi \in H^{1}(\Omega) .
$$

Now let $T: H^{1}(\Omega) \longrightarrow H^{-1}(\Omega)$ be the operator defined by

$$
\langle T u, \phi\rangle=\int_{\Omega} k_{\epsilon} \nabla u \nabla \phi+\int_{\Omega \backslash \omega_{\epsilon}} u^{3} \phi, \quad \forall \phi \in H^{1}(\Omega) .
$$

It is readily verified that $T$ is a potential operator, that is $T u-f$ is the derivative of the functional

$$
E(u)=\frac{1}{2} \int_{\Omega} k_{\epsilon}|\nabla u|^{2}+\frac{1}{4} \int_{\Omega \backslash \omega_{\epsilon}} u^{4}-\int_{\Omega} f u .
$$

Then, the theorem will follow by showing that $T$ is bounded, strictly monotone and coercive; in fact, by these properties of $T$ the functional $E$ is coercive and weakly lower semicontinuous on $H^{1}(\Omega)$ (see e.g. ${ }^{12}$, Theorem 26.11). Thus, $E$ is bounded from below and attains its infimum at some $u_{\epsilon} \in H^{1}(\Omega)$ satisfying $T u_{\epsilon}=f$. The uniqueness of $u_{\epsilon}$ is a consequence of the strict monotonicity of $T$; for, if $T u=T v=$ $f$, equation (4.3) below implies $u=v$.

i. $T$ is bounded.

By Hölder's inequality

$$
|\langle T u, \phi\rangle| \leq\|\nabla u\|_{L^{2}(\Omega)}\|\nabla \phi\|_{L^{2}(\Omega)}+\|u\|_{L^{6}(\Omega)}^{3}\|\phi\|_{L^{2}(\Omega)}
$$

and by Sobolev Embedding Theorem $\|u\|_{L^{6}(\Omega)} \leq C_{S}\|u\|_{H^{1}(\Omega)}$, so that

$$
\begin{aligned}
|\langle T u, \phi\rangle| & \leq\|\nabla u\|_{L^{2}(\Omega)}\|\nabla \phi\|_{L^{2}(\Omega)}+C_{S}^{3}\|u\|_{H^{1}(\Omega)}^{3}\|\phi\|_{L^{2}(\Omega)} \\
& \leq \max \left[\|u\|_{H^{1}(\Omega)}, C_{S}^{3}\|u\|_{H^{1}(\Omega)}^{3}\right]\|\phi\|_{H^{1}(\Omega)} .
\end{aligned}
$$

Therefore, if $u$ belongs to a bounded subset of $H^{1}(\Omega)$,

$$
\|T u\|_{H^{-1}(\Omega)}=\sup _{\phi} \frac{|\langle T u, \phi\rangle|}{\|\phi\|_{H^{1}(\Omega)}} \leq \max \left[\|u\|_{H^{1}(\Omega)}, C_{S}^{3}\|u\|_{H^{1}(\Omega)}^{3}\right]=C_{2} .
$$

ii. $T$ is (strictly) monotone.

In fact, we have

$$
\langle T u-T v, u-v\rangle=\int_{\Omega} k_{\epsilon}|\nabla(u-v)|^{2}+\int_{\Omega \backslash \omega_{\epsilon}}(u-v)^{2}\left(u^{2}+u v+v^{2}\right) \geq 0 .
$$

Furthermore

$$
\langle T u-T v, u-v\rangle=0 \quad \Leftrightarrow \quad u=v
$$

iii. $T$ is coercive, that is

$$
\lim _{\|u\|_{H^{1}(\Omega)} \rightarrow+\infty} \frac{\langle T u, u\rangle}{\|u\|_{H^{1}(\Omega)}}=+\infty .
$$


By using again Hölder's inequality,

$$
\begin{aligned}
\langle T u, u\rangle & \geq k \int_{\Omega}|\nabla u|^{2}+\int_{\Omega \backslash \omega_{\epsilon}} u^{4} \geq k\|\nabla u\|_{L^{2}(\Omega)}^{2}+\frac{1}{\left|\Omega \backslash \omega_{\epsilon}\right|}\left(\int_{\Omega \backslash \omega_{\epsilon}} u^{2}\right)^{2} \\
& \geq k\|\nabla u\|_{L^{2}(\Omega)}^{2}+\frac{1}{|\Omega|}\|u\|_{L^{2}\left(\Omega \backslash \omega_{\epsilon}\right)}^{4} \\
& =k\left(\|\nabla u\|_{L^{2}(\Omega)}^{2}+\|u\|_{L^{2}\left(\Omega \backslash \omega_{\epsilon}\right)}^{2}\right)+\frac{1}{|\Omega|}\|u\|_{L^{2}\left(\Omega \backslash \omega_{\epsilon}\right)}^{4}-k\|u\|_{L^{2}\left(\Omega \backslash \omega_{\epsilon}\right)}^{2} .
\end{aligned}
$$

Finally, by Poincaré inequality (see Appendix) and since $|\Omega|^{-1} x^{4}-k x^{2} \geq-k^{2}|\Omega| / 4$, we get

$$
\langle T u, u\rangle \geq k C\|u\|_{H^{1}(\Omega)}^{2}-\frac{k^{2}}{4}|\Omega|
$$

for some positive constant $C$; hence, (4.4) follows.

Remark 4.1. If $f$ is positive (i.e. $\langle f, \phi\rangle \geq 0$ for $\phi \geq 0$ ) it follows from (4.2) that $E(|u|) \leq E(u)$ for every $u \in H^{1}(\Omega)$; on the other hand, we proved in the previous theorem that $u_{\epsilon}$ is the unique minimum of $E$ in $H^{1}(\Omega)$. Then, we conclude that $u_{\epsilon} \geq 0$.

Remark 4.2. An alternative proof of theorem 4.1 can be obtained from the MintyBrowder Theorem (see e.g. ${ }^{8}$, Theorem 5.16 ) by showing that the (monotone, coercive) non linear operator $T$ is continuous. In fact, for $N \leq 3$ we have by Hölder inequality

$$
\begin{gathered}
\quad\left|\left\langle T u-T u_{0}, \phi\right\rangle\right|=\left|\int_{\Omega} k_{\epsilon} \nabla\left(u-u_{0}\right) \nabla \phi+\int_{\Omega \backslash \omega_{\epsilon}}\left(u-u_{0}\right)\left(u_{0}^{2}+u_{0} u+u^{2}\right) \phi\right| \\
\leq\left\|\nabla\left(u-u_{0}\right)\right\|_{L^{2}(\Omega)}\|\nabla \phi\|_{L^{2}(\Omega)}+\left\|u-u_{0}\right\|_{L^{6}(\Omega)}\left\|u_{0}^{2}+u_{0} u+u^{2}\right\|_{L^{3}(\Omega)}\|\phi\|_{L^{2}(\Omega)}
\end{gathered}
$$

for every $u_{0}, u, \phi$ in $H^{1}(\Omega)$. Hence, by the Sobolev embedding $H^{1}(\Omega) \hookrightarrow L^{6}(\Omega)$ we find that for every $u, u_{0}$ in a bounded subset of $H^{1}(\Omega)$ there exists a positive constant $K$ such that

$$
\left|\left\langle T u-T u_{0}, \phi\right\rangle\right| \leq K\left\|u-u_{0}\right\|_{H^{1}(\Omega)}\|\phi\|_{H^{1}(\Omega)}, \quad \forall \phi \in H^{1}(\Omega) .
$$

Then, it follows that $T$ is locally Lipschitz continuous.

\subsection{Main estimates}

In this section we will prove estimates on the solutions to (3.1) which will be useful in the subsequent discussion. To begin with, we have the following bound:

Proposition 4.1. Let $u_{\epsilon} \in H^{1}(\Omega)$ be a solution of (3.1). Then

$$
\left\|u_{\epsilon}\right\|_{H^{1}(\Omega)} \leq C\left(\|f\|_{H^{-1}}+\|f\|_{H^{-1}}^{3}\right)
$$

where $C=C(\Omega, k)$ is a positive constant. 
Proof. By putting $\phi=u=u_{\epsilon}$ in equation (4.1) and by definition (3.4), we readily get

$$
k\left\|\nabla u_{\epsilon}\right\|_{L^{2}(\Omega)}^{2}+\int_{\Omega \backslash \omega_{\epsilon}} u^{4} \leq\|f\|_{H^{-1}}\left\|u_{\epsilon}\right\|_{H^{1}(\Omega)} .
$$

By the above inequality we first obtain

$$
\left\|\nabla u_{\epsilon}\right\|_{L^{2}(\Omega)}^{2} \leq \frac{\|f\|_{H^{-1}}}{k}\left\|u_{\epsilon}\right\|_{H^{1}(\Omega)} .
$$

Furthermore, by the inequality

$$
\left\|u_{\epsilon}\right\|_{L^{2}\left(\Omega \backslash \omega_{\epsilon}\right)}^{4} \leq\left|\Omega \backslash \omega_{\epsilon}\right| \int_{\Omega \backslash \omega_{\epsilon}} u^{4} \leq|\Omega| \int_{\Omega \backslash \omega_{\epsilon}} u^{4}
$$

and again by (4.7) we get

$$
\left\|u_{\epsilon}\right\|_{L^{2}\left(\Omega \backslash \omega_{\epsilon}\right)}^{2} \leq\left(|\Omega|\|f\|_{H^{-1}}\left\|u_{\epsilon}\right\|_{H^{1}(\Omega)}\right)^{1 / 2} .
$$

Then, by using the Poincaré inequality (see Appendix)

$$
\begin{aligned}
\left\|u_{\epsilon}\right\|_{H^{1}(\Omega)}^{2} & \leq \frac{1}{C}\left(\left\|\nabla u_{\epsilon}\right\|_{L^{2}(\Omega)}^{2}+\left\|u_{\epsilon}\right\|_{L^{2}\left(\Omega \backslash \omega_{\epsilon}\right)}^{2}\right) \\
& \leq \frac{1}{k C}\|f\|_{H^{-1}}\left\|u_{\epsilon}\right\|_{H^{1}(\Omega)}+\frac{1}{C}\left(|\Omega|\|f\|_{H^{-1}}\right)^{1 / 2}\left\|u_{\epsilon}\right\|_{H^{1}(\Omega)}^{1 / 2} .
\end{aligned}
$$

We can write the above estimate in the form

$$
\left\|u_{\epsilon}\right\|_{H^{1}}^{1 / 2}\left(\left\|u_{\epsilon}\right\|_{H^{1}}-\frac{1}{k C}\|f\|_{H^{-1}}\right) \leq \frac{1}{C}|\Omega|^{1 / 2}\|f\|_{H^{-1}}^{1 / 2} .
$$

Now, either

$$
\left\|u_{\epsilon}\right\|_{H^{1}(\Omega)} \leq \frac{1}{k C}\|f\|_{H^{-1}}
$$

or

$$
\left(\left\|u_{\epsilon}\right\|_{H^{1}}-\frac{1}{k C}\|f\|_{H^{-1}}\right)^{3 / 2} \leq \frac{1}{C}|\Omega|^{1 / 2}\|f\|_{H^{-1}}^{1 / 2} .
$$

In both cases, we have that (4.6) holds.

Remark 4.3. We stress that for $\left|\omega_{\epsilon}\right| \rightarrow 0$ the constant $C$ appearing in inequalities (4.8) can be chosen independent of $\epsilon$ (see the discussion following equation (7.4) in the Appendix); hence, also the constant in the estimate (4.6) is independent of $\epsilon$.

Remark 4.4. By the above estimate and by the previously mentioned Sobolev embeddings, we obtain a priori bounds of the solutions in $L^{p}(\Omega)$, with $p \leq \frac{2 N}{N-2}$ if $N \geq 3$ and for every $p \geq 1$ if $N=2$.

One easily verifies that the bound (4.6) holds for the potential $U$ of the unperturbed problem (3.5) with $k=C=1$. We now prove additional properties of $U$ which will be useful in the sequel. 
Proposition 4.2. Let $\Omega$ be a bounded domain in $\mathbb{R}^{N}$ with $\partial \Omega \in C^{1,1}$ and let $f \in L^{p}(\Omega)$ for any $p>2$ if $N=2$ and for $p>3$ if $N=3$; then the (unique) weak solution $U$ of (3.5) satisfies

$$
\|U\|_{L^{\infty}(\Omega)},\|\nabla U\|_{L^{\infty}(\Omega)} \leq C\left(\|f\|_{L^{p}(\Omega)}+\|f\|_{L^{p}(\Omega)}^{3}\right) .
$$

Proof. By the previous remark, $U^{3} \in L^{p}(\Omega)$ for every $p \geq 1$ if $N=2$ and for $1 \leq p \leq 2$ if $N=3$; by the equation in (3.5) the same holds (in the weak sense) for $\Delta U$. Hence, we can apply known regularity results for the Neumann problem (see e.g., Theorem 2.4.2.7 in ${ }^{14}$ ) to conclude that $U$ belongs to $W^{2, p}(\Omega)$ for every $p>1$ if $N=2$ and for $1<p \leq 2$ if $N=3$, with

$$
\|U\|_{W^{2, p}(\Omega)} \leq C\left(\|f\|_{L^{p}(\Omega)}+\|f\|_{L^{p}(\Omega)}^{3}\right) .
$$

Now, it is known that $W^{2, p}(\Omega) \subset \mathcal{C}^{k}(\bar{\Omega})$ for $k=[2-N / p]$ (see ${ }^{8}$, section 9.3); hence, in the case $N=2$ it follows that $U \in \mathcal{C}^{1}(\bar{\Omega})$ whenever the datum $f$ in (3.5) satisfies $f \in L^{p}(\Omega)$ with $p>2$ and

$$
\|U\|_{L^{\infty}(\Omega)},\|\nabla U\|_{L^{\infty}(\Omega)} \leq C\left(\|f\|_{L^{p}(\Omega)}+\|f\|_{L^{p}(\Omega)}^{3}\right) .
$$

In the case $N=3$, one obtains that $U$ is Hölder continuous on $\Omega$; nevertheless, the same $\mathcal{C}^{1}$ regularity can be readily achieved by repeated application of the previous arguments since $U^{3}, f \in L^{p}(\Omega)$ with $p>3$ and hence $U \in W^{2, p}(\Omega)$ for $p>3$.

Let us now recall that for $f \geq 0$ we have $U \geq 0$ (see remark 4.1); furthermore, we have a comparison principle:

Proposition 4.3. Let $f_{2} \geq f_{1}$ satisfy the assumptions of Proposition 4.2 and let $U_{1}, U_{2}$ be the solutions to (3.5) with $f=f_{1}$ and $f=f_{2}$ respectively. Then, $U_{2} \geq U_{1}$ in $\Omega$.

Proof. The function $W=U_{2}-U_{1}$ solves the problem

$$
\begin{cases}-\Delta W=-Q W+f_{2}-f_{1} & \text { in } \Omega \\ \frac{\partial W}{\partial \mathbf{n}}=0, & \text { on } \partial \Omega,\end{cases}
$$

where $Q=U_{1}^{2}+U_{1} U_{2}+U_{2}^{2} \geq 0$. Let $\Omega^{-}=\{x \in \bar{\Omega} \mid W(x)<0\}$; since $W$ is continuous in $\bar{\Omega}$, the set $\Omega^{-}$is open. Moreover, by the above equation, $W$ is superharmonic in $\Omega^{-}$and therefore it assumes the minimum value at some point on the boundary $\partial \Omega^{-}$. On the other hand, such point must belong to $\overline{\partial \Omega^{-} \backslash \partial \Omega}$ due to the homogeneous Neumann condition and to the Hopf principle. But $W=0$ on this set, so that $\Omega^{-}=\emptyset$ and $W \geq 0$ in $\Omega$.

Corollary 4.1. Assume that $\operatorname{essinf}_{x \in \Omega} f(x)=m$. Then, the solution $U$ to problem (3.5) satisfies

$$
U(x) \geq m^{1 / 3}, \quad x \in \Omega .
$$


Proof. Apply Proposition 4.10 by choosing $f_{1}=m$ and $f_{2}=f$. Since $U_{1}=m^{1 / 3}$, the above bound follows.

Let us now discuss the regularity of the solution $u_{\epsilon}$ we first note that, by remark 4.4 , the term

$$
\chi_{\Omega \backslash \omega_{\epsilon}} u_{\epsilon}^{3}
$$

is bounded in $L^{p}(\Omega)$ for $1<p \leq 2$ if $N=3$ and in $L^{p}(\Omega)$ for any $p \geq 1$ if $N=2$. On the other hand, $u_{\epsilon}$ satisfies

$$
-\operatorname{div}\left(k_{\epsilon}(x) \nabla u_{\epsilon}\right)=f-\chi_{\Omega \backslash \omega_{\epsilon}} u_{\epsilon}^{3}, \quad x \in \Omega
$$

with $k_{\epsilon}$ defined by (3.4). Since $f \in L^{p}(\Omega)$ with $p>1$ if $N=2$ and $p>3$ if $N=3$ we can apply the interior estimate in ${ }^{13}$, Theorem 8.24 , which yields, for any $\Omega^{\prime} \subset \subset \Omega$

$$
\left\|u_{\epsilon}\right\|_{\mathcal{C}^{0, \alpha}\left(\bar{\Omega}^{\prime}\right)} \leq C\left(\left\|u_{\epsilon}\right\|_{L^{2}(\Omega)}+\left\|u_{\epsilon}\right\|_{L^{6}(\Omega)}^{3}+\|f\|_{L^{p}(\Omega)}\right) \leq C\left(\left\|u_{\epsilon}\right\|_{H^{1}(\Omega)}^{3}+\|f\|_{L^{p}(\Omega)}\right)
$$

where $0<\alpha<1, C>0$ only depend on $N, k, p$ and $\Omega^{\prime}$.

Finally, using (4.6), we obtain

$$
\left\|u_{\epsilon}\right\|_{\mathcal{C}^{0, \alpha}\left(\bar{\Omega}^{\prime}\right)} \leq C
$$

where $C$ depends only on $\Omega^{\prime}, k, N$ and on $\|f\|_{L^{p}(\Omega)}$. Now, by taking $\Omega^{\prime} \supset \omega_{\epsilon}$ and by observing that $k_{\epsilon}=1$ in $\Omega \backslash \Omega^{\prime}$, it is not difficult to show that $u_{\epsilon}$ is uniformly Hölder continuous in $\bar{\Omega}$ and that

$$
\left\|u_{\epsilon}\right\|_{\mathcal{C}^{0, \alpha}(\bar{\Omega})} \leq C
$$

where $C$ depends only on $\Omega, k, N$ and on $\|f\|_{L^{p}(\Omega)}$.

\subsection{Estimate on the $H^{1}$ norm of $u_{\epsilon}-U$}

Theorem 4.2. Let $f \in L^{p}(\Omega)$ for some $p>N(N=2,3)$; assume further that $f \geq m>0$ a.e. in $\Omega$. Let $U$ be the solution to problem (3.5) and $u_{\epsilon}$ the solution to problem (3.1). Then

$$
\left\|u_{\epsilon}-U\right\|_{H^{1}(\Omega)} \leq C\left|\omega_{\epsilon}\right|^{\frac{1}{2}}
$$

where $C$ is a positive constant that depends on $k, \Omega, m$ and on $\|f\|_{L^{p}(\Omega)}$.

Proof. Using (3.5), we obtain

$$
\begin{aligned}
-\Delta U & =-\operatorname{div}\left(k_{\epsilon} \nabla U\right)-\operatorname{div}\left(\left(1-k_{\epsilon}\right) \nabla U\right) \\
& =-\operatorname{div}\left(k_{\epsilon} \nabla U\right)-(1-k) \operatorname{div}\left(\chi_{\omega_{\epsilon}} \nabla U\right)=-U^{3}+f
\end{aligned}
$$

and therefore

$$
-\operatorname{div}\left(k_{\epsilon} \nabla U\right)+\chi_{\Omega \backslash \omega_{\epsilon}} U^{3}=f+(1-k) \operatorname{div}\left(\chi_{\omega_{\epsilon}} \nabla U\right)-\chi_{\omega_{\epsilon}} U^{3} .
$$


Now, subtracting the above (4.16) from the equation for $u_{\epsilon}$ in (3.1) we get

$$
-\operatorname{div}\left(k_{\epsilon} \nabla\left(u_{\epsilon}-U\right)\right)+\chi_{\Omega \backslash \omega_{\epsilon}}\left(u_{\epsilon}^{3}-U^{3}\right)=-(1-k) \operatorname{div}\left(\chi_{\omega_{\epsilon}} \nabla U\right)+\chi_{\omega_{\epsilon}} U^{3}
$$

that, letting $w_{\epsilon}=u_{\epsilon}-U$ and $q_{\epsilon}=U^{2}+U u_{\epsilon}+u_{\epsilon}^{2}$, we can rewrite as

$$
-\operatorname{div}\left(k_{\epsilon} \nabla w_{\epsilon}\right)+\chi_{\Omega \backslash \omega_{\epsilon}} w_{\epsilon} q_{\epsilon}=(k-1) \operatorname{div}\left(\chi_{\omega_{\epsilon}} \nabla U\right)+\chi_{\omega_{\epsilon}} U^{3} .
$$

Let us now observe that we can write $w_{\epsilon}=\tilde{w}_{\epsilon}+a_{\epsilon}$, where

$$
\int_{\Omega \backslash \omega_{\epsilon}} \tilde{w}_{\epsilon} q_{\epsilon}=0 \quad \text { and } \quad a_{\epsilon}=\frac{1}{\int_{\Omega \backslash \omega_{\epsilon}} q_{\epsilon}} \int_{\Omega \backslash \omega_{\epsilon}} w_{\epsilon} q_{\epsilon} .
$$

For the function $\tilde{w}_{\epsilon}$ we have by Poincaré inequality (see Appendix)

$$
\left\|\tilde{w}_{\epsilon}\right\|_{L^{2}} \leq C\left\|\nabla \tilde{w}_{\epsilon}\right\|_{L^{2}}\left(=C\left\|\nabla w_{\epsilon}\right\|_{L^{2}}\right) .
$$

Moreover, being

$$
\int_{\Omega} \operatorname{div}\left(k_{\epsilon} \nabla w_{\epsilon}\right)=\int_{\partial \Omega} \frac{\partial w_{\epsilon}}{\partial \mathbf{n}}=0
$$

from (3.5) and

$$
\int_{\Omega} \operatorname{div}\left(\chi_{\omega_{\epsilon}} \nabla U\right)=0
$$

from divergence theorem, using (4.17) and integrating over $\Omega$ we get

$$
\left|a_{\epsilon}\right|=\frac{1}{\int_{\Omega \backslash \omega_{\epsilon}} q_{\epsilon}}\left|\int_{\Omega \backslash \omega_{\epsilon}} w_{\epsilon} q_{\epsilon}\right|=\frac{1}{\int_{\Omega \backslash \omega_{\epsilon}} q_{\epsilon}}\left|\int_{\omega_{\epsilon}} U^{3}\right| .
$$

Now, by our assumptions on $f$, by the elementary estimate $q_{\epsilon} \geq \frac{3}{4} U^{2}$ and by (4.11), we readily obtain

$$
\left|a_{\epsilon}\right| \leq \frac{4}{3 m^{2 / 3}\left|\Omega \backslash \omega_{\epsilon}\right|}\|U\|_{L^{6}(\Omega)}^{3}\left|\omega_{\epsilon}\right|^{\frac{1}{2}} .
$$

Then, using (4.9), (4.19) and (4.21),

$$
\left\|w_{\epsilon}\right\|_{H^{1}}=\left\|\tilde{w}_{\epsilon}+a_{\epsilon}\right\|_{H^{1}} \leq\left\|\tilde{w}_{\epsilon}\right\|_{H^{1}}+\left|a_{\epsilon}\right||\Omega|^{\frac{1}{2}} \leq C\left(\left\|\nabla \tilde{w}_{\epsilon}\right\|_{L^{2}}+\left|\omega_{\epsilon}\right|^{\frac{1}{2}}\right) .
$$

We are now left to prove the estimate

$$
\left\|\nabla w_{\epsilon}\right\|_{L^{2}} \leq C\left|\omega_{\epsilon}\right|^{\frac{1}{2}} .
$$

To this aim, multiplying (4.17) times $w_{\epsilon}$ and integrating over $\Omega$ by parts, we get

$$
\int_{\Omega} k_{\epsilon}\left|\nabla w_{\epsilon}\right|^{2}+\int_{\Omega \backslash \omega_{\epsilon}} w_{\epsilon}^{2} q_{\epsilon}=-(k-1) \int_{\omega_{\epsilon}} \nabla U \nabla w_{\epsilon}+\int_{\omega_{\epsilon}} U^{3} w_{\epsilon}
$$

which leads to

$$
\begin{array}{r}
k\left\|\nabla w_{\epsilon}\right\|_{L^{2}}^{2} \leq\left|(1-k) \int_{\omega_{\epsilon}} \nabla U \nabla w_{\epsilon}\right|+\left|\int_{\omega_{\epsilon}} U^{3} w_{\epsilon}\right| \leq \\
\left\{(1-k)\|\nabla U\|_{L^{\infty}\left(\omega_{\epsilon}\right)}\left\|\nabla w_{\epsilon}\right\|_{L^{2}}+\|U\|_{L^{\infty}\left(\omega_{\epsilon}\right)}^{3}\left\|w_{\epsilon}\right\|_{L^{2}}\right\}\left|\omega_{\epsilon}\right|^{\frac{1}{2}} .
\end{array}
$$


using again the decomposition (4.18), Poincaré inequality (4.19) for $\tilde{w}_{\epsilon}$, estimate (4.21) for $a_{\epsilon}$ and (4.9) we obtain

$$
k\left\|\nabla w_{\epsilon}\right\|_{L^{2}}^{2} \leq C\left(\|f\|_{L^{p}(\Omega)}+\|f\|_{L^{p}(\Omega)}^{3}\right)\left\{\left\|\nabla w_{\epsilon}\right\|_{L^{2}}+\left|\omega_{\epsilon}\right|^{\frac{1}{2}}\right\}\left|\omega_{\epsilon}\right|^{\frac{1}{2}}
$$

where $C=C(k, \Omega)$.

Finally, solving second order inequality, we get

$$
\left\|\nabla w_{\epsilon}\right\|_{L^{2}} \leq C\left|\omega_{\epsilon}\right|^{\frac{1}{2}}
$$

where $C$ is a positive constant depending on $\Omega, k$ and on $\|f\|_{L^{p}(\Omega)}$. Then, by recalling (4.22), the theorem follows.

We now derive energy estimates for $u_{\epsilon}-U$.

Theorem 4.3. Let $f$ satisfy the same assumptions as in theorem 4.2. Then

$$
\left\|u_{\epsilon}-U\right\|_{L^{2}(\Omega)} \leq C\left|\omega_{\epsilon}\right|^{\frac{1}{2}+\eta}
$$

for some $\eta>0$ and where $C$ is a positive constant depending on $k, \Omega, m$ and on $\|f\|_{L^{p}(\Omega)}$.

Proof. Set $w_{\epsilon}=u_{\epsilon}-U$. Then, $w_{\epsilon} \in H^{1}(\Omega)$ satisfies

$\int_{\Omega} \nabla w_{\epsilon} \cdot \nabla \phi d x+\int_{\Omega \backslash \omega_{\epsilon}} q_{\epsilon} w_{\epsilon} \phi d x=(k-1) \int_{\omega_{\epsilon}} \nabla u_{\epsilon} \cdot \nabla \phi d x+\int_{\omega_{\epsilon}} U^{3} \phi d x \quad \forall \phi \in H^{1}(\Omega)$

where $q_{\epsilon}=u_{\epsilon}^{2}+u_{\epsilon}^{2} U^{2}+U^{2}$ and, by the estimate (4.15),

$$
\left\|w_{\epsilon}\right\|_{H^{1}(\Omega)} \leq C\left|\omega_{\epsilon}\right|^{1 / 2}
$$

where $C=C\left(k, \Omega,\|f\|_{L^{p}(\Omega)}\right)$. Consider now $\bar{w}_{\epsilon} \in H^{1}(\Omega)$ weak solution to

$$
\int_{\Omega} \nabla \bar{w}_{\epsilon} \cdot \nabla \phi d x+\int_{\Omega \backslash \omega_{\epsilon}} q_{\epsilon} \bar{w}_{\epsilon} \phi d x=\int_{\Omega} w_{\epsilon} \phi d x \quad \forall \phi \in H^{1}(\Omega) .
$$

Then, choosing $\phi=\bar{w}_{\epsilon}$, one has

$$
\left\|\bar{w}_{\epsilon}\right\|_{H^{1}(\Omega)} \leq C\left\|w_{\epsilon}\right\|_{H^{1}(\Omega)} \leq C\left|\omega_{\epsilon}\right|^{1 / 2} .
$$

Furthermore, by Theorem 2.4.2.7 in ${ }^{14}$ we have that $\bar{w}_{\epsilon}$ belongs to $H^{2}(\Omega)$

$$
\left\|\bar{w}_{\epsilon}\right\|_{H^{2}(\Omega)} \leq C\left\|w_{\epsilon}\right\|_{L^{2}(\Omega)} .
$$

Choosing $\phi=w_{\epsilon}$ into (4.27) we get

$$
\int_{\Omega} \nabla \bar{w}_{\epsilon} \cdot \nabla w_{\epsilon}, d x+\int_{\Omega \backslash \omega_{\epsilon}} q_{\epsilon} \bar{w}_{\epsilon} w_{\epsilon} d x=\int_{\Omega} w_{\epsilon}^{2} d x .
$$

On the other hand, choosing $\phi=\bar{w}_{\epsilon}$ into (4.26) we derive

$$
\int_{\Omega} \nabla \bar{w}_{\epsilon} \cdot \nabla w_{\epsilon}, d x+\int_{\Omega \backslash \omega_{\epsilon}} q_{\epsilon} \bar{w}_{\epsilon} w_{\epsilon} d x=(k-1) \int_{\omega_{\epsilon}} \nabla u_{\epsilon} \cdot \nabla \bar{w}_{\epsilon} d x+\int_{\omega_{\epsilon}} U^{3} \bar{w}_{\epsilon} d x
$$


Hence, by (4.29) and (4.30), we have

$$
\int_{\Omega} w_{\epsilon}^{2} d x=(k-1) \int_{\omega_{\epsilon}} \nabla u_{\epsilon} \cdot \nabla \bar{w}_{\epsilon} d x+\int_{\omega_{\epsilon}} U^{3} \bar{w}_{\epsilon} d x .
$$

From (4.28) and Sobolev Embedding Theorem we have that $\bar{w}_{\epsilon} \in W^{1, p^{\prime}}\left(\omega_{\epsilon}\right)$ for any $p^{\prime}>1$ if $N=2$ and for $1<p^{\prime} \leq 6$ if $N=3$

$$
\left\|\bar{w}_{\epsilon}\right\|_{W^{1, p^{\prime}}(\Omega)} \leq C\left\|w_{\epsilon}\right\|_{L^{2}(\Omega)} .
$$

Since $U \in H^{1}(\Omega)$, again from Sobolev Embedding Theorem, $U^{3} \in L^{p}$, for all $p>1$ if $N=2$ and for $1<p \leq 2$ if $N=3$. Hence, applying Holder inequality and choosing $1<p<2$, we get

$$
\int_{\Omega} w_{\epsilon}^{2} d x \leq|k-1|\left\|\nabla \bar{w}_{\epsilon}\right\|_{L^{p^{\prime}}\left(\omega_{\epsilon}\right)}\left\|\nabla u_{\epsilon}\right\|_{L^{p}\left(\omega_{\epsilon}\right)}+\left\|\bar{w}_{\epsilon}\right\|_{L^{p^{\prime}}\left(\omega_{\epsilon}\right)}\left\|U^{3}\right\|_{L^{p}\left(\omega_{\epsilon}\right)} .
$$

Observe now that

$$
\left\|\nabla u_{\epsilon}\right\|_{L^{p}\left(\omega_{\epsilon}\right)} \leq\left\|\nabla w_{\epsilon}\right\|_{L^{p}\left(\omega_{\epsilon}\right)}+\|\nabla U\|_{L^{p}\left(\omega_{\epsilon}\right)} .
$$

By (4.9) the second term can be bounded as follows

$$
\|\nabla U\|_{L^{p}\left(\omega_{\epsilon}\right)} \leq C\left(\|f\|_{L^{p}(\Omega)}+\|f\|_{L^{p}(\Omega)}^{3}\right)\left|\omega_{\epsilon}\right|^{1 / p}
$$

where $C=C(\Omega)$. Moreover, by Hölder inequality and by the energy estimates (4.15) we have

$$
\left\|\nabla w_{\epsilon}\right\|_{L^{p}\left(\omega_{\epsilon}\right)} \leq\left|\omega_{\epsilon}\right|^{\frac{1}{p}-\frac{1}{2}}\left\|w_{\epsilon}\right\|_{H^{1}} \leq C\left|\omega_{\epsilon}\right|^{1 / p} .
$$

Hence, we get the bound

$$
\left\|\nabla u_{\epsilon}\right\|_{L^{p}\left(\omega_{\epsilon}\right)} \leq C\left|\omega_{\epsilon}\right|^{1 / p}
$$

where $C=C\left(\|f\|_{L^{p}(\Omega)}, k, m\right)$. Analogously

$$
\left\|U^{3}\right\|_{L^{p}\left(\omega_{\epsilon}\right)} \leq C\left|\omega_{\epsilon}\right|^{1 / p}
$$

where $C=C\left(\Omega,\|f\|_{L^{p}(\Omega)}\right)$. Recalling (4.32), we get

$$
\int_{\Omega} w_{\epsilon}^{2} \leq C\left\|w_{\epsilon}\right\|_{L^{2}(\Omega)}\left|\omega_{\epsilon}\right|^{1 / p}
$$

which finally gives

$$
\left\|w_{\epsilon}\right\|_{L^{2}(\Omega)} \leq C\left|\omega_{\epsilon}\right|^{1 / p}
$$

with $\frac{1}{p}>\frac{1}{2}$ and $C=C\left(k,\left(\|f\|_{L^{p}(\Omega)}\right)\right.$. 


\section{Proof of main result: the asymptotic formula}

In this section we deduce an asymptotic representation formula for the perturbed potential

$$
w_{\epsilon}=u_{\epsilon}-U
$$

analogous to the one obtained in Theorem 1 of ${ }^{9}$ for a voltage perturbation in the presence of inhomogeneities.

Let $N_{U}(x, y)$ be the Neumann function of the operator $-\Delta+3 U^{2}$ with homogeneous Neumann condition defined in (3.6). Note that we can write

$$
N_{U}(x, y)=N(x, y)+z(x, y)
$$

where $N$ is the Neumann function for the Laplacian, satisfying

$$
-\Delta_{x} N(x, y)=\delta(x-y) \quad \text { for } x \in \Omega,\left.\quad \frac{\partial N}{\partial n_{x}}\right|_{\partial \Omega}=\frac{1}{|\partial \Omega|}
$$

and, for every $y \in \Omega$, the function $x \mapsto z(x, y)$ solves the problem

$$
\begin{cases}-\Delta_{x} z(x, y)+3 U^{2}(x) z(x, y)=-3 U^{2}(x) N(x, y) & \text { in } \Omega \\ \frac{\partial z}{\partial n_{x}}=-\frac{1}{|\partial \Omega|}, & \text { on } \partial \Omega .\end{cases}
$$

We recall that $N(\cdot, y) \in W^{1,1}(\Omega)$ and therefore it belongs to $L^{p}(\Omega)$ for $p$ in some interval depending on the dimension (for every $p>1$ in dimension two). Then, by the smoothness of $U$ and by the same regularity arguments as in the previous section, we may take $z$ and $\nabla z$ continuous and bounded; it follows in particular that

$$
N_{U}(\cdot, y) \in L^{p}(\Omega) .
$$

Let us now multiply both the equations (3.1) and (3.5) by a test function $\phi$, integrate by parts and use the boundary condition; we get the identity

$$
\int_{\Omega} k_{\epsilon} \nabla u_{\epsilon} \nabla \phi+\int_{\Omega \backslash \omega_{\epsilon}} u_{\epsilon}^{3} \phi=\int_{\Omega} \nabla U \nabla \phi+\int_{\Omega} U^{3} \phi
$$

By subtracting to both sides of (5.3) the quantity

$$
\int_{\Omega} \nabla u_{\epsilon} \nabla \phi+\int_{\Omega} u_{\epsilon}^{3} \phi
$$

we obtain

$$
\int_{\omega_{\epsilon}}(k-1) \nabla u_{\epsilon} \nabla \phi-\int_{\omega_{\epsilon}} u_{\epsilon}^{3} \phi=\int_{\Omega} \nabla\left(U-u_{\epsilon}\right) \nabla \phi+\int_{\Omega}\left(U^{3}-u_{\epsilon}^{3}\right) \phi .
$$

By introducing the perturbed potential $w_{\epsilon}=u_{\epsilon}-U$, we can write the above equation in the form

$$
\int_{\Omega} \nabla w_{\epsilon} \nabla \phi+\int_{\Omega} w_{\epsilon}\left(U^{2}+U u_{\epsilon}+u_{\epsilon}^{2}\right) \phi=\int_{\omega_{\epsilon}}(1-k) \nabla u_{\epsilon} \nabla \phi+\int_{\omega_{\epsilon}} u_{\epsilon}^{3} \phi .
$$


Finally, by using the identity

$$
U^{2}+U u_{\epsilon}+u_{\epsilon}^{2}=3 U^{2}+3 U w_{\epsilon}+w_{\epsilon}^{2}
$$

we get

$$
\int_{\Omega} \nabla w_{\epsilon} \nabla \phi+\int_{\Omega} 3 U^{2} w_{\epsilon} \phi=\int_{\omega_{\epsilon}}(1-k) \nabla u_{\epsilon} \nabla \phi+\int_{\omega_{\epsilon}} u_{\epsilon}^{3} \phi-\int_{\Omega} 3 U w_{\epsilon}^{2} \phi-\int_{\Omega} w_{\epsilon}^{3} \phi .
$$

Let us fix $y \in \partial \Omega$ (or even $y \in \Omega \backslash \bar{\omega}_{\epsilon}$ ) and let $\phi_{m} \in \mathcal{C}^{1}(\Omega)$ be a sequence converging to $N_{U}(\cdot, y)$ in $W^{1,1}(\Omega)$ and in $\mathcal{C}^{1}(\bar{D})$, where $\omega_{\epsilon} \subset D \subset \subset \Omega$. Now, the regularity of $U$ provided by (4.9) and the discussion following (4.12) allow us to insert $\phi_{m}$ into (5.4) and to pass to the limit, so that

$$
\begin{aligned}
\int_{\Omega} \nabla w_{\epsilon} \nabla_{x} N_{U} d x+\int_{\Omega} 3 U^{2} w_{\epsilon} N_{U} d x & =\int_{\omega_{\epsilon}}(1-k) \nabla u_{\epsilon} \nabla_{x} N_{U} d x+\int_{\omega_{\epsilon}} u_{\epsilon}^{3} N_{U} d x \\
& -\int_{\Omega} 3 U w_{\epsilon}^{2} N_{U} d x-\int_{\Omega} w_{\epsilon}^{3} N_{U} d x
\end{aligned}
$$

After integration by parts in the first term by using (3.6) (here we exploit the homogeneous Neumann condition satisfied by $N_{U}$ ) we obtain

$$
w_{\epsilon}(y)=\int_{\omega_{\epsilon}}(1-k) \nabla u_{\epsilon} \nabla_{x} N_{U} d x+\int_{\omega_{\epsilon}} u_{\epsilon}^{3} N_{U} d x-\int_{\Omega} 3 U w_{\epsilon}^{2} N_{U} d x-\int_{\Omega} w_{\epsilon}^{3} N_{U} d x .
$$

The following result is a first step towards an asymptotic representation formula in our non linear setting:

Proposition 5.1. Let $\chi_{\omega_{\epsilon}}$ denote the indicator function of the set $\omega_{\epsilon}$. Then the following relation holds

$$
w_{\epsilon}(y)=\left|\omega_{\epsilon}\right|\left((1-k) \int_{\Omega}\left|\omega_{\epsilon}\right|^{-1} \chi_{\omega_{\epsilon}} \nabla u_{\epsilon} \nabla_{x} N_{U} d x+\int_{\Omega}\left|\omega_{\epsilon}\right|^{-1} \chi_{\omega_{\epsilon}} u_{\epsilon}^{3} N_{U} d x\right)+o\left(\left|\omega_{\epsilon}\right|\right) .
$$

Proof. We need to prove suitable bounds of the two last terms in (5.5). By Hölder inequality, the last term is bounded by $\left\|w_{\epsilon}\right\|_{L^{3 q}(\Omega)}^{3}\left\|N_{U}\right\|_{L^{p}(\Omega)}$, where $q=p /(p-1)$. Hence, by Sobolev embedding and by (4.15) we get

$$
\left|\int_{\Omega} w_{\epsilon}^{3} N_{U} d x\right| \leq C\left|\omega_{\epsilon}\right|^{3 / 2}
$$

for some constant $C$ depending on $k, \Omega$ and $U$. Let us now consider the remaining term; by the boundedness of $U$ and again by Hölder inequality we have

$$
\left|\int_{\Omega} 3 U w_{\epsilon}^{2} N_{U} d x\right| \leq 3\|U\|_{L^{\infty}(\Omega)}\left|\int_{\Omega} w_{\epsilon}^{2} N_{U} d x\right| \leq 3\|U\|_{L^{\infty}(\Omega)}\left\|N_{U}\right\|_{L^{p}(\Omega)}\left\|w_{\epsilon}\right\|_{L^{2 q}(\Omega)}^{2} .
$$


By a version of the Gagliardo-Nirenberg inequality for bounded domains (the constants depending only on $q$ and on the domain, see ${ }^{18}$ ) we now get

$$
\begin{aligned}
\left\|w_{\epsilon}\right\|_{L^{2 q}(\Omega)} \leq C_{1} \| \nabla & w_{\epsilon}\left\|_{L^{2}(\Omega)}^{1-\frac{1}{q}}\right\| w_{\epsilon}\left\|_{L^{2}(\Omega)}^{\frac{1}{q}}+C_{2}\right\| w_{\epsilon} \|_{L^{2}(\Omega)} \\
& \leq \tilde{C}_{1}\left|\omega_{\epsilon}\right|^{\frac{1}{2}+\frac{\eta}{q}}+\tilde{C}_{2}\left|\omega_{\epsilon}\right|^{\frac{1}{2}+\eta} .
\end{aligned}
$$

Then, the proposition follows by inserting these estimates into (5.5).

Remark 5.1. Equation (5.6) should be compared with the analogous formula (8) given in ${ }^{9}$ for the steady state voltage perturbation caused by internal conductivity inhomogeneities. The different sign of the term containing the gradients is due to the definition (3.6) of the Green function $N_{U}$.

Following ${ }^{9}$ we now introduce the variational solutions $V^{(j)}, v_{\epsilon}^{(j)}$ to the problems

$$
\begin{cases}\Delta V^{(j)}=0 & \text { in } \Omega \\ \frac{\partial V^{(j)}}{\partial \mathbf{n}}=n_{j} & \text { on } \partial \Omega\end{cases}
$$

and

$$
\begin{cases}\operatorname{div}\left(k_{\epsilon}(x) \nabla v_{\epsilon}^{(j)}\right)=0 & \text { in } \Omega \\ \frac{\partial v_{\epsilon}^{(j)}}{\partial \mathbf{n}}=n_{j} & \text { on } \partial \Omega\end{cases}
$$

$n_{j}$ being the $j$-th coordinate of the outward normal to $\partial \Omega$ and where the functions $V^{(j)}, v_{\epsilon}^{(j)}$ are normalized by $\int_{\partial \Omega} V^{(j)}=\int_{\partial \Omega} v_{\epsilon}^{(j)}=0$. We observe that

$$
V^{(j)}=x_{j}-\frac{1}{|\partial \Omega|} \int_{\partial \Omega} x_{j}
$$

and that the difference $v_{\epsilon}^{(j)}-V^{(j)}$ satisfies estimates analogous to (4.15) and to (4.25) ( see $^{9}$ sect.2). Hence, by integration by parts and by exploiting such estimates, we get ( see $^{9}$ sect.3, eqs. (20)-(21))

$$
\begin{aligned}
& \int_{\Omega} k_{\epsilon} \nabla\left(u_{\epsilon}-U\right) \nabla\left(v_{\epsilon}^{(j)} \phi\right) d x \\
& =\int_{\Omega} \nabla\left(u_{\epsilon}-U\right) \nabla\left(V^{(j)} \phi\right) d x+\int_{\omega_{\epsilon}}(k-1) \nabla\left(u_{\epsilon}-U\right) \nabla \phi V^{(j)} d x+o\left(\left|\omega_{\epsilon}\right|\right)
\end{aligned}
$$

for every $\phi$ smooth enough. Now, again using the weak form of the equations (3.1) and (3.5), we easily get the identities

$$
\begin{aligned}
\int_{\Omega} k_{\epsilon} \nabla\left(u_{\epsilon}-U\right) \nabla\left(v_{\epsilon}^{(j)} \phi\right) d x & =\int_{\omega_{\epsilon}}(1-k) \nabla U \nabla\left(v_{\epsilon}^{(j)} \phi\right) d x \\
& +\int_{\omega_{\epsilon}} U^{3} v_{\epsilon}^{(j)} \phi d x+\int_{\Omega \backslash \omega_{\epsilon}}\left(U^{3}-u_{\epsilon}^{3}\right) v_{\epsilon}^{(j)} \phi d x \\
\int_{\Omega} \nabla\left(u_{\epsilon}-U\right) \nabla\left(V^{(j)} \phi\right) d x & =\int_{\omega_{\epsilon}}(1-k) \nabla u_{\epsilon} \nabla\left(V^{(j)} \phi\right) d x \\
+ & \int_{\omega_{\epsilon}} U^{3} V^{(j)} \phi d x+\int_{\Omega \backslash \omega_{\epsilon}}\left(U^{3}-u_{\epsilon}^{3}\right) V^{(j)} \phi d x .
\end{aligned}
$$


By inserting these into (5.11) we obtain

$$
\begin{aligned}
\int_{\omega_{\epsilon}}(1-k) & \nabla U \nabla\left(v_{\epsilon}^{(j)} \phi\right) d x+\int_{\omega_{\epsilon}} U^{3} v_{\epsilon}^{(j)} \phi d x+\int_{\Omega \backslash \omega_{\epsilon}}\left(U^{3}-u_{\epsilon}^{3}\right) v_{\epsilon}^{(j)} \phi d x \\
= & \int_{\omega_{\epsilon}}(1-k) \nabla u_{\epsilon} \nabla\left(V^{(j)} \phi\right) d x+\int_{\omega_{\epsilon}} U^{3} V^{(j)} \phi d x+\int_{\Omega \backslash \omega_{\epsilon}}\left(U^{3}-u_{\epsilon}^{3}\right) V^{(j)} \phi d x \\
& +\int_{\omega_{\epsilon}}(k-1) \nabla\left(u_{\epsilon}-U\right) \nabla \phi V^{(j)} d x+o\left(\left|\omega_{\epsilon}\right|\right)
\end{aligned}
$$

that is, by straightforward rearrangements,

$$
\begin{aligned}
(1-k) & \int_{\omega_{\epsilon}} \nabla U \nabla\left(v_{\epsilon}^{(j)} \phi\right) d x \\
= & (1-k)\left[\int_{\omega_{\epsilon}} \nabla u_{\epsilon} \nabla\left(V^{(j)} \phi\right) d x-\int_{\omega_{\epsilon}} \nabla u_{\epsilon} \nabla \phi V^{(j)} d x+\int_{\omega_{\epsilon}} \nabla U \nabla \phi V^{(j)} d x\right] \\
& -\int_{\omega_{\epsilon}} U^{3}\left(v_{\epsilon}^{(j)}-V^{(j)}\right) \phi d x+\int_{\Omega \backslash \omega_{\epsilon}}\left(u_{\epsilon}^{3}-U^{3}\right)\left(v_{\epsilon}^{(j)}-V^{(j)}\right) \phi d x+o\left(\left|\omega_{\epsilon}\right|\right) .
\end{aligned}
$$

By the boundedness of $U, u_{\epsilon}$, by Hölder inequality and by the previous $L^{2}$ estimates of the perturbations $u_{\epsilon}-U$ and $v_{\epsilon}^{(j)}-V^{(j)}$, we conclude that the whole last term of the above equation is $o\left(\left|\omega_{\epsilon}\right|\right)$. Hence we can write

$$
\begin{aligned}
& \int_{\omega_{\epsilon}} \nabla U \nabla\left(v_{\epsilon}^{(j)} \phi\right) d x \\
& =\int_{\omega_{\epsilon}} \nabla u_{\epsilon} \nabla\left(V^{(j)} \phi\right) d x-\int_{\omega_{\epsilon}} \nabla u_{\epsilon} \nabla \phi V^{(j)} d x+\int_{\omega_{\epsilon}} \nabla U \nabla \phi V^{(j)} d x+o\left(\left|\omega_{\epsilon}\right|\right) \\
& =\int_{\omega_{\epsilon}} \nabla u_{\epsilon} \nabla V^{(j)} \phi d x+\int_{\omega_{\epsilon}} \nabla U \nabla \phi V^{(j)} d x+o\left(\left|\omega_{\epsilon}\right|\right) \\
& =\int_{\omega_{\epsilon}} \nabla u_{\epsilon} \nabla V^{(j)} \phi d x+\int_{\omega_{\epsilon}}^{(j)} \nabla U \nabla \phi v_{\epsilon}^{(j)} d x \\
& \quad+O\left(\left\|v_{\epsilon}^{(j)}-V^{(j)}\right\|_{L^{2}(\Omega)}\left|\omega_{\epsilon}\right|^{1 / 2}\|\nabla U\|_{L^{\infty}\left(\omega_{\epsilon}\right)}\right)+o\left(\left|\omega_{\epsilon}\right|\right) \\
& =\int_{\omega_{\epsilon}} \nabla u_{\epsilon} \nabla V^{(j)} \phi d x+\int_{\omega_{\epsilon}} \nabla U \nabla \phi v_{\epsilon}^{(j)} d x+o\left(\left|\omega_{\epsilon}\right|\right) .
\end{aligned}
$$

After a further rearrangement, we get

$$
\int_{\omega_{\epsilon}} \nabla U \nabla v_{\epsilon}^{(j)} \phi d x=\int_{\omega_{\epsilon}} \nabla u_{\epsilon} \nabla V^{(j)} \phi d x+o\left(\left|\omega_{\epsilon}\right|\right) .
$$

A final rescaling yields

$$
\int_{\Omega} \nabla U\left|\omega_{\epsilon}\right|^{-1} \chi_{\omega_{\epsilon}} \nabla v_{\epsilon}^{(j)} \phi d x=\int_{\Omega}\left|\omega_{\epsilon}\right|^{-1} \chi_{\omega_{\epsilon}} \nabla u_{\epsilon} \nabla V^{(j)} \phi d x+o(1) .
$$

For sake of clarity from now on we will use the component notation rather than the matrix notation. Moreover the convention of summation over repeated indices is used. By the results in ${ }^{9}$ there exist a regular Borel measure $\mu$, functions $\mathcal{M}_{i j} \in$ $L^{2}(\Omega, d \mu)$ and a sequence $\omega_{\epsilon_{n}}$, with $\left|\omega_{\epsilon_{n}}\right| \rightarrow 0$, such that

$$
\left|\omega_{\epsilon_{n}}\right|^{-1} \chi_{\omega_{\epsilon_{n}}} d x \rightarrow d \mu, \quad\left|\omega_{\epsilon_{n}}\right|^{-1} \chi_{\omega_{\epsilon_{n}}} \frac{\partial}{\partial x_{i}} v_{\epsilon_{n}}^{(j)} d x \rightarrow \mathcal{M}_{i j} d \mu
$$


in the weak* topology of the dual of $\mathcal{C}^{0}(\bar{\Omega})$. Then, passing to the limit in (5.12) and by recalling (5.10) we can state

Proposition 5.2. Let $u_{\epsilon}, U$ denote the solutions to (3.1) and (3.5) and let $\omega_{\epsilon_{n}}$, with $\left|\omega_{\epsilon_{n}}\right| \rightarrow 0$, be a sequence satisfying (3.2) and (5.13). Then

$$
\lim _{n \rightarrow \infty}\left|\omega_{\epsilon_{n}}\right|^{-1} \chi_{\omega_{\epsilon_{n}}} \frac{\partial u_{\epsilon_{n}}}{\partial x_{j}} d x=\mathcal{M}_{i j} \frac{\partial U}{\partial x_{i}} d \mu
$$

in the weak ${ }^{*}$ topology of the dual of $\mathcal{C}^{0}(\bar{\Omega})$.

We are now in position to prove our asymptotic representation formula. We will state it here in a more precise way:

Theorem 5.1. Let $u_{\epsilon}, U$ denote the solutions to (3.1) and (3.5) and let $\omega_{\epsilon_{n}}$, with $\left|\omega_{\epsilon_{n}}\right| \rightarrow 0$, be a sequence satisfying (3.2) and (5.13). Then, if $w_{\epsilon_{n}}=u_{\epsilon_{n}}-U$, we have

$$
w_{\epsilon_{n}}(y)=\left|\omega_{\epsilon_{n}}\right| \int_{\Omega}\left((1-k) \mathcal{M}_{i j} \frac{\partial U}{\partial x_{i}} \frac{\partial N_{U}}{\partial x_{j}}+U^{3} N_{U}\right) d \mu(x)+o\left(\left|\omega_{\epsilon_{n}}\right|\right) \quad y \in \partial \Omega
$$

where $N_{U}(x, y)$ is the solution of (3.6).

Proof. By proposition 5.1 we have

$$
\begin{array}{r}
w_{\epsilon_{n}}(y)=\left|\omega_{\epsilon_{n}}\right|\left(\int_{\Omega}(1-k)\left|\omega_{\epsilon_{n}}\right|^{-1} \chi_{\omega_{\epsilon_{n}}} \nabla u_{\epsilon_{n}} \nabla_{x} N_{U} d x+\right. \\
\left.\int_{\Omega}\left|\omega_{\epsilon_{n}}\right|^{-1} \chi_{\omega_{\epsilon_{n}}} u_{\epsilon_{n}}^{3} N_{U} d x\right)+o\left(\left|\omega_{\epsilon_{n}}\right|\right)
\end{array}
$$

Let $K_{0}$ is a compact set such that $\omega_{\epsilon} \subset K_{0} \subset \Omega$. By the properties of $N_{U}$ we can find a vector valued test function $\Phi_{y} \in \mathcal{C}^{0}(\bar{\Omega})$ such that

$$
\Phi_{y}(x)=\nabla_{x} N_{U}(x, y), \quad \text { for } x \in K_{0}, y \in \partial \Omega .
$$

Then, by proposition 5.2 ,

$$
\int_{\Omega}(1-k)\left|\omega_{\epsilon_{n}}\right|^{-1} \chi_{\omega_{\epsilon_{n}}} \frac{\partial u_{\epsilon_{n}}}{\partial x_{j}} \frac{\partial N_{U}}{\partial x_{j}} d x=\int_{\Omega}(1-k) \mathcal{M}_{i j} \frac{\partial U}{\partial x_{i}} \frac{\partial N_{U}}{\partial x_{j}} d \mu(x)+o(1) .
$$

Moreover, by now standard estimates one can readily prove

$$
\int_{\Omega}\left|\omega_{\epsilon_{n}}\right|^{-1} \chi_{\omega_{\epsilon_{n}}} u_{\epsilon_{n}}^{3} N_{U} d x=\int_{\Omega} U^{3} N_{U} d \mu(x)+o(1) .
$$

By inserting the above relations in the previous identity, the theorem follows.

We are now ready for:

Proof of theorem 3.1. The asymptotic formula (3.7) is the same as equation (5.15) proved in the previous theorem. In order to prove the last statement of the theorem, we remark that the polarization tensor $\mathcal{M}_{i j}$ is defined exactly as in ${ }^{9}$; hence, the 
stated properties follow by the same arguments as in section 4 of ${ }^{9}$ with trivial modifications.

Remark 5.2. It is well known, see e.g. ${ }^{4}$, that the polarization tensor $\mathcal{M}=$ $\mathcal{M}(k, D)$ can be computed explicitly for particular shapes of $D$. For instance, in the case of a ball, the polarization tensor takes the form $\mathcal{M}=\frac{2}{1+k}|D| \mathbf{I}$ where $\mathbf{I}$ is the identity matrix. If $D$ is an ellipse whose focal line is on either the $x$-axis or the $y$-axis, its semi-major axis is of length $a$ and its semi-minor axis is of length $b=e a$, then its polarization matrix is given by

$$
\mathcal{M}=|D|\left|\begin{array}{cc}
\frac{1+e}{1+k e} & 0 \\
0 & \frac{1+e}{e+k}
\end{array}\right|
$$

Remark 5.3. We would like to emphasize that, with minor changes, the asymptotic expansion generalizes to the case of anisotropic conductivities of the form

$$
\mathcal{K}_{\epsilon}= \begin{cases}K_{1} \text { in } & \Omega \backslash \omega_{\epsilon} \\ K_{2} \text { in } \omega_{\epsilon}\end{cases}
$$

where $K_{1}, K_{2}$ are symmetric constant matrices satisfying

$$
|\xi|^{2} \leq \xi^{T} K_{1} \xi \leq \beta_{1}|\xi|^{2}, \quad \forall \xi \in \mathbb{R}^{2},
$$

and

$$
\alpha_{2}|\xi|^{2} \leq \xi^{T} K_{2} \xi \leq \beta_{2}|\xi|^{2}, \quad \forall \xi \in \mathbb{R}^{2}
$$

with $\beta_{1} \geq 1$ and $0<\alpha_{2} \leq \beta_{2}<1$. The asymptotic formula then reads as follows

$$
\left.w_{\epsilon}(y)=\int_{\Omega} \mathcal{M}_{i j}\left(K_{1}-K_{2}\right)_{i k} \frac{\partial U}{\partial x_{k}} \frac{\partial N_{U}}{\partial x_{j}}+U^{3} N_{U}\right)+o\left(\epsilon^{N}\right) \quad y \in \partial \Omega,
$$

where $N_{U}(x, y)$ is the Neumann function of the operator $-\operatorname{div}\left(K_{1} \nabla \cdot\right)+3 U^{2}$ with homogeneous Neumann conditions.

\section{Localization of small inhomogeneities}

Let us consider the case of a finite number of well separated inhomogeneities of small diameter $\epsilon$ centered at points $z_{1}, \ldots, z_{m} \in \Omega$ that we assume of the form $z_{l}+\epsilon D_{l}, l=1, \ldots, m$ with conductivities $0<k_{l}<1, l=1, \ldots, m$. As $\epsilon \rightarrow 0$, one obtains from the asymptotic formula (3.7) (see also ${ }^{9}$ ) the following formula, valid for any $y \in \partial \Omega$ :

$$
w_{\epsilon}(y)=\epsilon^{N} \sum_{l=1}^{m}\left(\left(1-k_{l}\right) \mathcal{M}_{i j}^{l}\left(z_{l}\right) \frac{\partial U}{\partial x_{i}}\left(z_{l}\right) \frac{\partial N_{U}}{\partial x_{j}}\left(z_{l}, y\right)+U^{3}\left(z_{l}\right) N_{U}\left(z_{l}, y\right)\right)+o\left(\epsilon^{N}\right) .
$$


We now show a possible use of the derived asymptotic formula, together with a suitable integration of (measured) boundary data, to obtain useful identities for localizing the inhomogeneities and reconstructing the polarization tensor.

Let $g \in H^{-1 / 2}(\partial \Omega)$ be a given function and consider the (unique) solution $W \in H^{1}(\Omega)$ of the boundary value problem

$$
\begin{cases}-\Delta W+3 U^{2} W=0 & \text { in } \Omega \\ \frac{\partial W}{\partial \mathbf{n}}=g & \text { on } \partial \Omega,\end{cases}
$$

where $U$ is the background potential which solves (3.5). Then,

$$
W(z)=\int_{\partial \Omega} N_{U}(z, y) g(y) d S_{y} \quad z \in \Omega
$$

where $N_{U}$ is the Neumann function defined by (3.6). The proof follows readily by observing that, due to the homogeneous Neumann condition satisfied by $N_{U}$, we can write

$$
\int_{\partial \Omega} N_{U}(z, y) g(y) d S_{y}=\int_{\partial \Omega}\left(N_{U}(z, y) \frac{\partial W}{\partial \mathbf{n}}-\frac{\partial N_{U}}{\partial \mathbf{n}}(z, y) W(y)\right) d S_{y} .
$$

We now consider the average measurement

$$
\Gamma \equiv \int_{\partial \Omega} w_{\epsilon}(y) g(y) d S_{y}
$$

By inserting (6.1) in this expression

$$
\begin{aligned}
\Gamma=\epsilon^{N} \sum_{l=1}^{m}\left[\int _ { \partial \Omega } \left(\left(1-k_{l}\right) \mathcal{M}_{i j}^{l}\left(z_{l}\right) \frac{\partial U}{\partial x_{i}}\left(z_{l}\right) \frac{\partial N_{U}}{\partial x_{j}}\left(z_{l}, y\right)\right.\right. \\
\left.\left.+U^{3}\left(z_{l}\right) N_{U}\left(z_{l}, y\right)\right) g(y) d S_{y}\right]+o\left(\epsilon^{N}\right)
\end{aligned}
$$

and taking account of (6.3) we have proved the following

Proposition 6.1. Let $u_{\epsilon}$ be the solution to (3.1) with inhomogeneities $z_{l}+\epsilon D_{l}$, conductivities $0<k_{l}<1$ and polarization tensors $\mathcal{M}_{i j}^{l}$ for $l=1, \ldots, m$. Let $U$ denote the solutions to (3.5) and let $W$ be solution of (6.2). Then,

$$
\Gamma=\epsilon^{N} \sum_{l=1}^{m}\left[\left(1-k_{l}\right) \mathcal{M}_{i j}^{l}\left(z_{l}\right) \frac{\partial U}{\partial x_{i}}\left(z_{l}\right) \frac{\partial W}{\partial x_{j}}\left(z_{l}\right)+U^{3}\left(z_{l}\right) W\left(z_{l}\right)\right]+o\left(\epsilon^{N}\right) .
$$

We can apply the previous formula to the simple case of approximating the location and the polarization tensor of a single small inhomogeneity (in two dimensions) centered at the point $(\bar{x}, \bar{y})$. Our approach will consist of two steps: we first detect the position of the inhomogeneity by considering a constant background potential, by solving problem (6.2) for suitable choices of the boundary data and inserting the solution $W$ into (6.5); subsequently, in the simple case of a rectangular domain, we construct examples of the auxiliary function $W$ corresponding to admissible non constant background potentials, which allow to recover the matrix elements of the polarization tensor 
We start by determining the location of the inhomogeneity. We observe that by choosing a constant datum $f$ in problem (3.5), the (unique) solution is a constant background potential $U=\lambda \equiv f^{1 / 3}$. In that case, the equation for the auxiliary function $W$ becomes

$$
-\Delta W(x, y)+3 \lambda^{2} W(x, y)=0 .
$$

The above equation has a family of solutions of the form

$$
W(x, y)=e^{a x+b y}, \quad a, b \in \mathbb{R}
$$

provided that $a^{2}+b^{2}=3 \lambda^{2}$. In particular, we have the two solutions

$$
W_{1}(x, y)=e^{\lambda \sqrt{3} x}, \quad W_{2}(x, y)=e^{\lambda \sqrt{3} y}
$$

respectively with Neumann data

$$
g_{1}(x, y)=\lambda \sqrt{3} n_{1}(x, y) e^{\lambda \sqrt{3} x}, \quad g_{2}(x, y)=\lambda \sqrt{3} n_{2}(x, y) e^{\lambda \sqrt{3} y}, \quad(x, y) \in \partial \Omega
$$

where $n_{i}, i=1,2$ are the component of the normal unit vector to $\partial \Omega$.

Now, let $u_{\epsilon}$ be the solution of (3.1) with $f=\lambda^{3}$ and denote by $\Gamma_{1}(\lambda), \Gamma_{2}(\lambda)$ the average measurements (6.4) with $w_{\epsilon}=u_{\epsilon}-\lambda$ and boundary data $g_{1}, g_{2}$, respectively; we obtain from (6.5) (with $N=2, m=1$ )

$$
\Gamma_{1}(\lambda)=\epsilon^{2} \lambda^{3} e^{\lambda \sqrt{3} \bar{x}}+o\left(\epsilon^{2}\right), \quad \Gamma_{2}(\lambda)=\epsilon^{2} \lambda^{3} e^{\lambda \sqrt{3} \bar{y}}+o\left(\epsilon^{2}\right) .
$$

By choosing a specific value of $\lambda$, the above relations can be used to approximate the position of the center of a small inhomogeneity.

Next we determine the polarization tensor. Its determination requires a non constant background potential $U$. In order to further simplify the problem, we assume that $\Omega=[0,1] \times[0,1]$ and try to identify the single element $M_{11}$ of the $(2 \times 2)$ polarization matrix (we also assume that $k$ is known). By the geometry of the domain, we can take a background potential $U=U(x)$ independent of $y$, provided that $U^{\prime}(0)=U^{\prime}(1)=0$. Hence, we look for an auxiliary function $W=W(x)$ which solves the linear ordinary equation of the second order

$$
-W^{\prime \prime}(x)+3 U(x)^{2} W(x)=0 .
$$

By looking for a solution in the form

$$
W(x)=e^{\varphi(x)}
$$

we find that the function $\varphi$ satisfies the equation

$$
\varphi^{\prime \prime}(x)+\varphi^{\prime}(x)^{2}=3 U(x)^{2} .
$$

By the substitution $\psi(x)=\varphi^{\prime}(x)$ we are reduced to a first order Riccati equation

$$
{ }^{\prime}(x)+\psi(x)^{2}=3 U(x)^{2}
$$

In general, there are no explicit solutions of such equation for a given $U$; on the other hand, there are large families of functions $\psi$ such that the left hand side of (6.7) is a positive function with vanishing derivative at $x=0$ and $x=1$. Thus, for 
any such $\psi$, the function $U$ defined (except for the sign) by (6.7) is an admissible background potential. For example, a straightforward calculation shows that

$$
\psi(x)=\frac{1}{3}\left(x^{2}+x-3\right)
$$

solves (6.7) with $U(x)$ smooth function in $[0,1]$ satisfying homogeneous Neumann conditions (it can be easily seen that no linear $\psi$ can generate an admissible non constant potential). Then, if we have previously detected the position $(\bar{x}, \bar{y})$, the matrix element $M_{11}$ can be approximated by (i) inserting in the right hand side of (6.5) the values $U(\bar{x}), U^{\prime}(\bar{x})$ calculated with the above potential, together with $W(\bar{x})=e^{\int \psi(\bar{x})}, W^{\prime}(\bar{x})=\psi(\bar{x}) e^{\int \psi(\bar{x})}$; note that by an appropriate choice of the integration constant we may take $e^{\int \psi(\bar{x})}=1$. Then, (ii) putting at the left hand side of (6.5) the average measurement (6.4) with $g$ the Neumann datum of $W(x)$ and $w_{\epsilon}=u_{\epsilon}-U, u_{\epsilon}=u_{\epsilon}(x, y)$ being the solution of (3.1) with $f(x, y)=-U^{\prime \prime}(x)+U(x)^{3}$. In order to detect $M_{22}$, we can proceed in a similar way, by taking the auxiliary function $W=W(y)$ and the related background potential $U=U(y)$.

Remark 6.1. It may be interesting to compare the above discussion to the detection of one small inhomogeneity for the linear problem in ${ }^{3}$. We stress that the reconstruction algorithm for the non linear problem, though more difficult from a computational point of view, allows to detect the position of the inhomogeneity (by using a constant background potential) independently of the polarization tensor. However, it is not clear if it is possible to perform an efficient localization of many separated inhomogeneities.

\section{Appendix: Poincaré inequalities}

There are different versions of inequalities which are usually known as Poincaré inequalities. Essentially, they relate the $L^{2}$ norm of the fluctuation of a function to the $L^{2}$ norm of its gradient. In this paper we use the following special case of the inequality proved in ${ }^{15}$, Theorem 8.11 :

Theorem 7.1. Let $g$ be a function in $L^{2}(\Omega)$ such that $\int_{\Omega} g=1$. Then, there is $S>0$ which depends on $\Omega, g$, such that for any $u \in H^{1}(\Omega)$

$$
\left\|u-\int_{\Omega} u g\right\|_{L^{2}(\Omega)} \leq S\|\nabla u\|_{L^{2}(\Omega)} .
$$

The proof follows a classical reductio ad absurdum argument relying on compactness.

If we now choose $u \equiv u_{\epsilon}$,

$$
g=\left|\Omega \backslash \omega_{\epsilon}\right|^{-1} \chi_{\Omega \backslash \omega_{\epsilon}}
$$

and put

$$
\bar{u}_{\epsilon}=\left|\Omega \backslash \omega_{\epsilon}\right|^{-1} \int_{\Omega \backslash \omega_{\epsilon}} u_{\epsilon}
$$


we obtain

$$
\left\|u_{\epsilon}\right\|_{L^{2}(\Omega)}^{2} \leq 2\left(\left\|u_{\epsilon}-\bar{u}_{\epsilon}\right\|_{L^{2}(\Omega)}^{2}+|\Omega| \bar{u}_{\epsilon}^{2}\right) \leq 2 S^{2}\left\|\nabla u_{\epsilon}\right\|_{L^{2}(\Omega)}^{2}+2 \frac{|\Omega|}{\left|\Omega \backslash \omega_{\epsilon}\right|}\left\|u_{\epsilon}\right\|_{L^{2}\left(\Omega \backslash \omega_{\epsilon}\right)}^{2} .
$$

By this estimate it follows easily

$$
\left\|u_{\epsilon}\right\|_{H^{1}(\Omega)}^{2} \leq \frac{1}{C}\left(\left\|\nabla u_{\epsilon}\right\|_{L^{2}(\Omega)}^{2}+\left\|u_{\epsilon}\right\|_{L^{2}\left(\Omega \backslash \omega_{\epsilon}\right)}^{2}\right)
$$

which was used in theorem 4.1 and in proposition 4.1. Since the functions (7.2) are uniformly bounded for $\epsilon \rightarrow 0$, one can show that the costant $S$ can be chosen independent of $\epsilon$; thus, by (7.3), we can also take $C$ independent of $\epsilon$ in (7.4).

Finally, by choosing $u \equiv w_{\epsilon}=u_{\epsilon}-U$,

$$
g=\left(\int_{\Omega \backslash \omega_{\epsilon}} q_{\epsilon}\right)^{-1} q_{\epsilon} \chi_{\Omega \backslash \omega_{\epsilon}}
$$

(where $q_{\epsilon}=U^{2}+U u_{\epsilon}+u_{\epsilon}^{2}$ ) and by recalling (4.18), we readily see that (7.1) is equivalent to the estimate (4.19).

\section{References}

1. R.R. Aliev and A.V. Panfilov, A simple two-variable model of cardiac excitation, Chaos, Solitons \& Fractals 7(3), 1996, 293-301.

2. D. Alvarez, F.A. Atienza, J.L. Rojo-Alvarez, A. García-Alberola and M. Moscoso, Shape reconstruction of cardiac ischemia from non-contact intracardiac recordings: a model study, Math. Comput. Modelling 55, 2012, 1770-1781.

3. H. Ammari, S. Moskow and M. Vogelius, Boundary integral formulae for the reconstruction of electric and electromagnetic inhomogeienities of small volume, ESAIM, Control, Optimisation and Calculus of Variations 9, 2003, 49-66.

4. H. Ammari and H. Kang, Reconstruction of small inhomogeneities from boundary measurements, Springer, Lectures Notes in Mathematics Series, vol. 1846, 2004.

5. M. Boulakia, S. Cazeau, M.A Fernández, J.F. Gerbeau and N. Zemzemi, Mathematical Modeling of Electrocardiograms: A Numerical Study, Annals of Biomedical Engineering 38(3), 2010, 1071-1097.

6. M. Boulakia, M.A Fernández, J.F. Gerbeau and N. Zemzemi, A coupled system of PDEs and ODEs arising in electrocardiograms modeling, Applied Math. Res. Exp., 2008(abn002), 28, 2008.

7. M. Boulakia, J.F. Gerbeau and E. Schenone, Reduced-order modeling for cardiac electrophysiology. Application to parameter identification, Int. J. Numer. Methods Engng 28, 2012, 727-744.

8. H. Brezis, Functional Analysis, Sobolev Spaces and Partial Differential Equations, Springer, 2011.

9. Y. Capdeboscq and M. Vogelius, A general representation formula for boundary voltage perturbations caused by internal conductivity inhomogeneities of low volume fraction, Math. Modelling and Num. Analysis 37, 2003, 159-173.

10. P. Colli Franzone, B. Taccardi and C. Viganotti, An approach to inverse calculation of epicardial potentials from body surface maps, Adv. Cardiol. 21, 1978, 50-54.

11. P. Colli Franzone, L.F. Pavarino and S. Scacchi, Mathematical cardiac electrophysiology, Springer-Verlag Italia, Milano, Modeling, Simulation and Applications (MS\&A) Series, vol. 13, 2014. 
12. S. Fucik and A. Kufner, Nonlinear Differential Equations, Elsevier, 1980.

13. D. Gilbarg and N.S. Trudinger, Elliptic Partial Differential Equations of Second Order, Springer Verlag, Berlin, 1983.

14. P. Grisvard, Elliptic problems in nonsmooth domains, Monographs and Studies in Mathematics, 24. Pitman (Advanced Publishing Program), Boston, MA, 1985.

15. E.H. Lieb and M. Loss, Analysis - second edition, Graduate Studies in Mathematics vol. 14, American Mathematical Society, 2001.

16. M. Lysaker, B.F. Nielsen, Towards a level set framework for infarction modeling: an inverse problem, Int. Journal of Numerical Analysis and Modeling 3(4), 2006, 377-394.

17. B.F. Nielsen, M. Lysaker and A. Tveito, On the use of the resting potential and level set methods for identifying ischemic heart disease: an inverse problem, J. Comput. Phys. 220, 2007, 772-790.

18. L. Nirenberg, On elliptic partial differential equations, Annali della Scuola Norm. Sup. - Pisa, Classe di Scienze, Sér. 3, 13(2), 1959, 115-162.

19. A.J. Pullan, L.K. Cheng, M.P. Nash, A. Ghodrati, R. MacLeod and D.H. Brooks, The inverse problem of electrocardiography. In Comprehensive Electrocardiology, P.W. Macfarlane, A. van Oosterom, O. Pahlm, P. Kligfield, M. Janse and J. Camm (Eds.), 299-344, Springer London, 2010.

20. J. Sundes, G.T. Lines, X. Cai, B.F. Nielsen, K.A. Mardal and A. Tveito, Computing the electrical activity in the heart, Springer, Monographs in Computational Science and Engineering Series, vol. 1, 2006.

21. Y. Yamashita, Theoretical studies on the inverse problem in electrocardiography and the uniqueness of the solution IEEE Trans. Biomed. Engng. BME-29(11), 1982, 719725 . 